\title{
Obtaining Personal Figure Mannequin Using Two Photographs for Three-Dimensional Corsetry Design
}

\author{
Nadezhda KORNILOVA ${ }^{1}$, Anna GORELOVA ${ }^{1,2}$, Dmitry VASILYEV ${ }^{2}$ \\ ${ }^{1}$ Ivanovo State Polytechnic University, Ivanovo, Russia; \\ 2 IIT Consulting Ltd, Ivanovo, Russia \\ http://dx.doi.org/10.15221/14.321
}

\begin{abstract}
:
In designing of corsetry the essential feature is figure's torso and breast form and size setting. Personal figure digital mannequins, obtained by the most 3D Body Scanning technologies, don't provide the ability to modify the shape of the breast and monitor the preservation of its main parameters during model design.

We have developed a mannequin mathematical model and an adjustment method to recover personal torso and breast geometry. For making breast simulated model, setting of different figure types, either in brassieres of different designs, or without them, methods of geometric and physical modeling were used. As a geometric model the part of ellipsoid was chosen, which represented a rotary body, formed by certain function graphics. For obtaining ellipsoid forms which are typical for the large breast size and for women of the older age group, physical modeling was used. Eight different parameters, sufficient to generate all possible forms of breast, are defined.

Our adjusting procedure needs only two photographs and 6 measured values.

Shape of torso is adjusted automatically using outlines deposited on the pictures, and values of the chest, waist and hips girths (Fig.2). Breast shape is adjusted interactively by changing the values of 8 parameters of the mathematical model (Fig.3). These parameters can be used further for modeling of brassier effect on the breast when 3-dimension corset wear construction is made.

Adjusting process takes less than 5 minutes, after which the designer can create a model of the corset, draw seams, reflect the properties of materials (Fig.5) and receive a set of patterns automatically, without any usage of various methods of receiving patterns. Advantages of our program are visualization of clothes appearance, availability of fitting it on the individual figure (Fig.6) and automated algorithm to convert three-dimensional details of clothes in the patterns.
\end{abstract}

Keywords: virtual mannequin, breast, geometric and physical modeling of breast's model, patterns of close-fitted clothes.

\section{Introduction}

CAD development in the area of sewing garment design in three-dimensional graphic software environment is one of the most actual sphere of researching, highly developed nowadays. Undoubted advantages of such programs are visualization of product appearance, availability of trying it on the individual figure and CAD of synthesizing type, automated algorithm of detail scanning construction, without any usage of various design methods [1-5]. In that case the quality of the good depends on specification accuracy of a starting 3-D model design directly. But the existing base of virtual mannequins of the main CAD is not sufficient, suggested means of parameter changing are not matched to the methods of size feature measurement used in design standards of different clothes types.

On-line sales growth of clothes in Internet requires the necessity of individual virtual mannequin development. Besides the mannequin setting to customer individual parameters with the help of the existing mathematical CAD is quite a routine and tiresome process, which doesn't guarantee the high quality result.

In designing corset wares it is not only important to have a well-defined breast surface with an accurate breast bottom replacement, but it is preferable to have the opportunity of modeling its changes with the help of corset wares, to be more concrete waist line tightening, breast form changing or shifting it up saving its volume.

In the majority of CAD the mannequin surface is represented as a single geometrical surface and has irregular triangular network structure which reduces the accuracy of anthropometrical breast point position determination, and the methods of correction don't assure the changing of breast form with its unchangeable volume. It is also related to the individual mannequins, made with the help of body scanning. 
The aim of this work is the development of virtual figure mannequin design method with a detail breast examination and setting it to the individual figure sizes.

In designing of breast models the methods of parametrical construction were chosen, because only they can combine all the majority of the breast forms, either in brassieres of different designs, or without them. For developing methods of obtaining individual figure mannequins the preferences were given to digital photographs as the most available means in communicating with a customer through the Internet. Digital photographs contain full information about torso form (about bearing, the main levels replacement, waist form, hips form and etc.), about breast form, and the customer appearance, which is very important in choosing the colour of the model.

\section{Theoretical researches}

\subsection{Method of virtual mannequin making for corset ware design}

In is very difficult to make a single parametrical construction of 3-D figure form and breast shape because of the variety of breast forms and some problems in their description in detail examination. The virtual mannequin surface suggested by us contains two models: breast model and torso model. They are independent algorithmically, which gives the opportunity of designing breast model of different varieties. The torso surface on the chest area has a form of the chest.

The mannequin torso represents the digital model, stated in the form of 3-D coordinate list of key points grouped in horizontal cross-sections:

$$
V_{o}=\left\{x_{o}, y_{o}, z_{o}\right\}
$$

Basic model dimensional form is obtained by scanning of female figure mannequin sculpture standard for the corset ware design. It includes 17 horizontal cross-sections with from 4 to 8 points in each.

The mannequins of other sizes are made with the help of geometrical transformations of a basic model. The starting point for mannequin changing is key point coordinates transformation matrix $\Delta \mathrm{V}=\{\mathrm{dx}, \mathrm{dy}, \mathrm{dz}\}$, determining the changes of the main size features in $1 \mathrm{sm}$ without any changes in semantical integrity and at the same time with saving elasticity and smoothness of the object.

Imperfection of key point coordinates transformation on different mannequin levels generates a need for setting of a main size feature in any cross-section. For the corset ware such size features are "Girth under breast -Gub", "Waist girth- Gw", "Hips girth- Gh" and "Height - P". The matrix $f=\left\{f_{\Gamma}, f_{T}\right.$, $\left.f_{6}, f_{p}\right\}$ is used for revealing of each key point identity toward a main size feature. The elements of this matrix may have 0 magnitude (not depending on this size) or may have magnitude $=1$ (the main size ). The process of other size mannequin obtaining includes the determination of a new key point replacement:

$$
V_{c}=V_{o}+\Delta V\left(f_{r}\left(G u b_{c}-G u b_{0}\right)+f_{T}\left(G w_{c}-G w_{0}\right)+f_{r}\left(G h_{c}-G h_{0}\right)+f_{p}\left(P_{c}-P_{0}\right)\right) .
$$

So we can assure the construction of any size variant mannequin of typical body structure with a step of any size feature change (Gub, Gw, Gh, P) in $1 \mathrm{sm}$.

For making breast simulated model, methods of geometric and physical modeling were used [6]. As a geometric model the part of ellipsoid was chosen, which represented a rotary body, formed by certain function graphics.

For providing the opportunity to obtain different breast forms within the limits of one mathematic model for rotary body generatrix, the function given below was chosen

$$
y=f(x, D, H, n, m)
$$

where

- $\quad D$ and $H$ set ellipsoid size and they are suitable to general breast size characteristic features: $d_{2 \varkappa}$ (horizontal diameter) and $H$ (convexity);

- $\quad n$ and $m$ determine generatrix form at the vertex and at the bottom of rotary body accordingly. For obtaining natural drooping which are typical for the large breast size and for women of the older age group, physical modeling was used. In so far as the amount of muscle tissue in the breast is insignificant, its form is defined by gravitation force and resistance to tension balance, i.e. it depends on its size and hypodermic layer elasticity. For the calculation of ellipsoid top shifting 
under the influence of its own weight, the method of sections used in theoretical mechanics was applied. For this purpose geometric model was divided into $h$ parts with a step of $\Delta h$ (Fig. 1, a). In accordance with it the parts of ellipsoid were cut off, starting with the bottom, changing it with force $F_{i}$, which magnitude is corresponding to weight $P_{i}$, cut off part(Fig. 1, b). Force $F_{i}$ produces the shifting of this section concerning to given form to the magnitude $\Delta z_{i}$ (Fig. 1, C). This magnitude according to the Hook's law may be defined as:

$$
\Delta z_{i}=f\left(\rho, G, S_{i} ; \Delta h\right)
$$

where $\rho$ - is breast density;

$G$ - is a shift modulus, depending on skin elasticity;

$\Delta h$ - is a step of mesh;

$S_{i}$ - is a area of vertical $i$-section of ellipsoid.

Maximum relative shift can be seen at the bottom of ellipsoid, and maximum absolute shift can be seen in the nipple point $\Delta z_{c m}$ (Fig. 1, d).

Shift of the concrete figure nipple point $\Delta z_{c m}$ may be defined with the help of size feature magnitude the points of its base $B \kappa 2$ and its bottom Boz.

As a result of the carried out calculations, it was stated that the magnitude $G$ changes from 50 to $2500 \mathrm{H} / \mathrm{m}^{2}$. The magnitude $\mathrm{G} \leq 500 \mathrm{H} / \mathrm{m}^{2}$ corresponds to "drooping" breast form, and $\mathrm{G} \geq 1500 \mathrm{H} / \mathrm{m}^{2}$ is more typical for cup breast form.

This parameter can be used for modeling of brassier effect on the breast when 3-dimension corset wear construction is made. For this purpose it is enough to increase the magnitude $G$ taking into account the design effect of brassiere cups and straps.

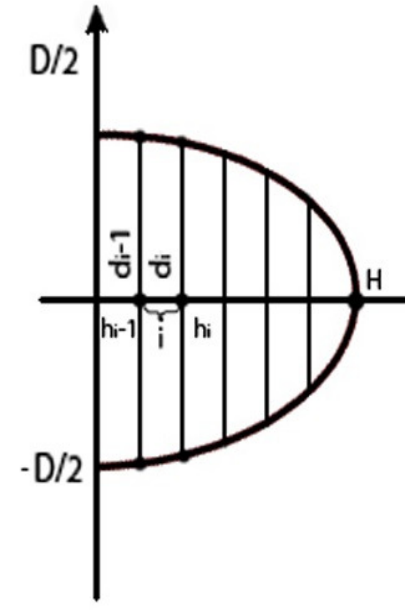

a

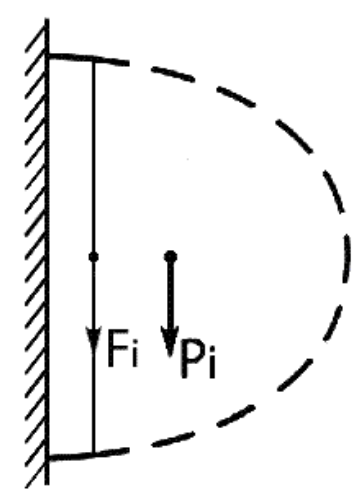

b

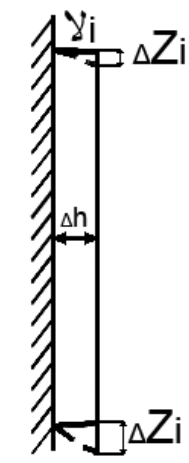

C

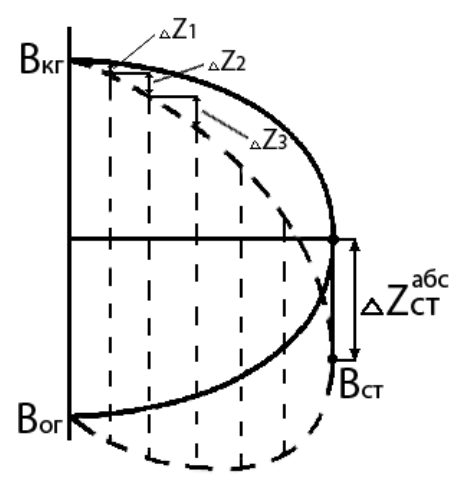

d

Fig. 1. Explanation to breast physical model

\subsection{Method of virtual mannequin adjustment according to individual parameters}

Ability of mannequin adjustment according to concrete figure anthropomorphic data is provided by the usage of generating model concept, intending the basic model construction with the set component of dimensional form $\mathrm{V}_{\mathrm{o}}$. A basic model guarantees anatomic naturalness, adequate plastics, topology and mannequin surface aesthetics. The mannequin surface is regarded as a higher-order non-linear surface, stated by hybrid parametrical model. Polygon surface remains unchangeable (or some insignificant changes are available) in its topology, and algorithmic part of model is used for simulating of anatomical features in the whole range of in-parameters.

For a basic model adjustment according to individual figure torso shape the full face photographs of customer figure, profile photographs and three main size features (girth under breast, waist girth, hips girth) are used. Using customers photographs in interactive mode (applying spline lines over the figure delineation) the replacement of the most characteristic front-back and side mannequin points are defined. The program makes mannequin transformation (shift of point group in a plane of section) with proportion changes subjected to surface smoothness. Then section scaling of size feature are carried out. During this process the adjusted basic model is subjected to compression 
strains-tensile strains deformation of some section parts in such a way that it can fulfill the set of restrictions and the objective function presenting deformation magnitude of starting model can take the minimal magnitude. For this purpose mannequin change algorithm for every group of independent parameters (girths) regarding to anatomical check-points (key points) is used. Its aim is to adjust an accurate parameter magnitude at the exact part of measurement. It allows to change a mannequin according to the setting parameters keeping smoothness, aesthetic plastics and anthropomorphic surface.

The developed algorithm allows to use the customer photograph of any scale, without any special demands to the background quality and additional calibration means, which gives the opportunity to more convenient communication between a designer and a customer through the Internet.

The next stage is a determination of breast shape in the brassiere, which is carried out with the help of interactive parameter setting of developed mathematical breast model:

1. "vertical position" changes breast bottom level,

2. "horizontal position" and "angle of turn" of breast corresponding to mid-sagittal plane allows to set central, middle and lateral breast position without changing its size,

3. "radius" defines transverse diameter of breast,

4. "protrusion" adjusts a level of beast protrusion regarding to the chest,

5. "elastic coefficient" allows to regulate breast protruding point regarding to the breast bottom and to design natural bottom part "drooping" in case of drop-shaped and bulb-shaped breast forms,

6. "roundness degree" allows to change a profile form of breast central part from cone shaped to spherical ones,

7. "sphericity degree" allows to change breast bottom form from smoothed to sharp-outlined.

Using these settings you can succeed in making accurate similarity of a breast form and a size in the brassiere. Conformity estimation of figure sizes is made with the help of CAD of the main breast size features (vertical arch, horizontal arch, distance between nipples, breast horizontal diameter, distance between medial points) and tools for measurements of mannequin girths at any level.

\section{Results}

The results of the theoretical researches were used in special CAD for 3-D corset wares BustCAD (Ivanovo, Russia). The program has a comfortable interface and a base of typical size variants corresponding to standard corset ware measurements, which gives the opportunity to obtain the desired shape, torso size and breast size within 2-5 minutes.

The form changing of virtual mannequin in designing of a new corset ware model is made by means of 8 parameter value specifications in the menu "Breast". The stages of virtual mannequin adjustment to an individual figure on the photograph are shown on fig. 2 and fig. 3.

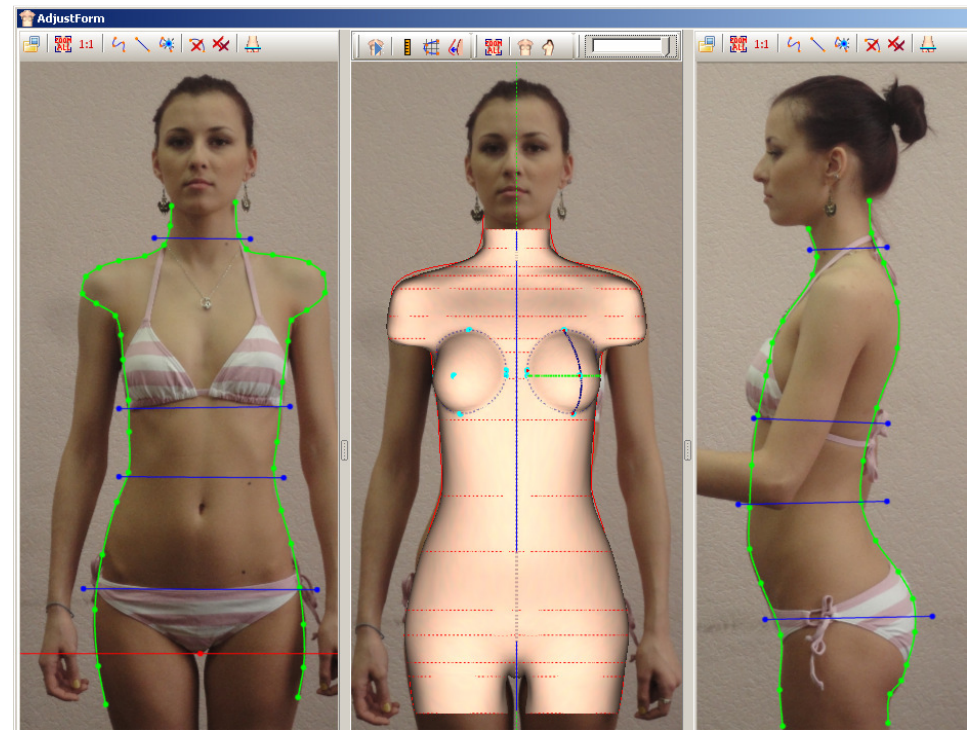

Fig. 2. Applying of delineation lines on the photograph and torso form adjustment

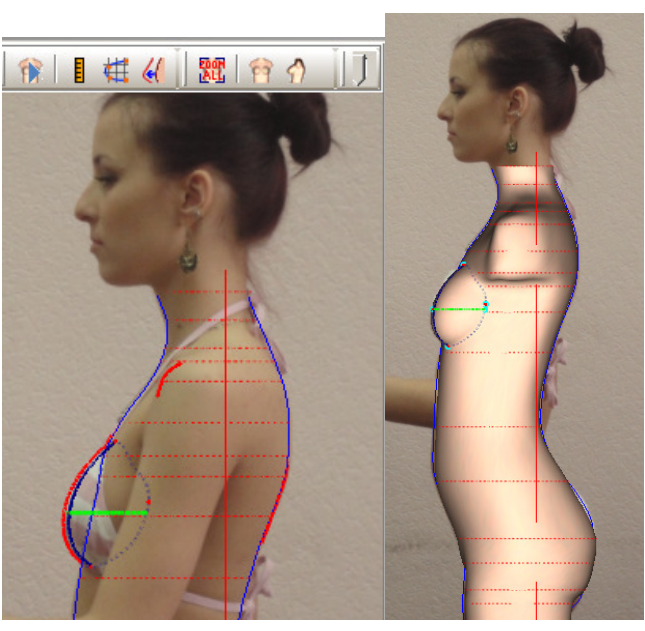

Fig. 3. Breast shape adjustment by changing of mathematical model parameters 
Quality estimation of mannequin adjustment to individual figure parameters on the examples of 50 women of different size groups was made. As a result it was stated that relative deviation of the main size features is in the permissible limits (See tabl. 1, fig. 4).

Table 1. Relative deviation in sizes of virtual mannequin and real figure in design corset ware

\begin{tabular}{|l|c|}
\hline \multicolumn{1}{|c|}{ Name of size feature } & Relative deviation, \% \\
\hline The third breast girth & 0,1 \\
\hline Breast centre & 1,8 \\
\hline Cross arc & 2,1 \\
\hline Vertical arc & 2,5 \\
\hline Distance between median points & 0 \\
\hline Waist girth & 0,1 \\
\hline Hips girth & 0,2 \\
\hline
\end{tabular}
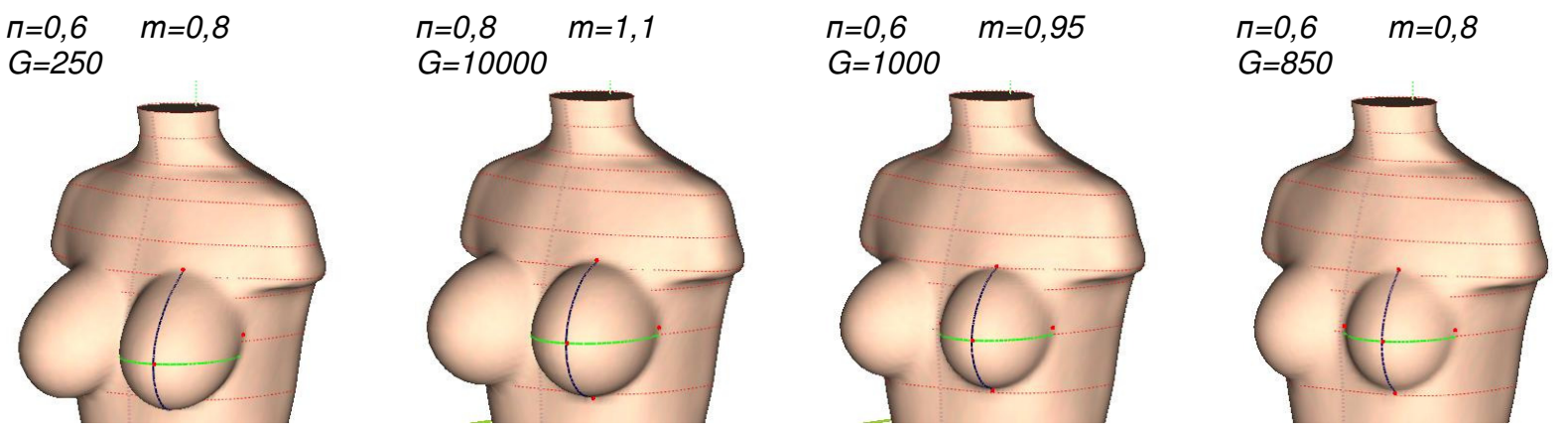

Figure 4. Virtual mannequin variants

Further model design in BustCAD consists of model lines applying directly on the mannequin surface and grouping of these lines in cutting detail. The material pattern may be projected on the each detail surface which gives the opportunity to value ware appearance. The program allows to reflect 3-D form of designed model directly on the figure photograph to estimate the image created by designer and consumer (Fig.5, fig. 6).

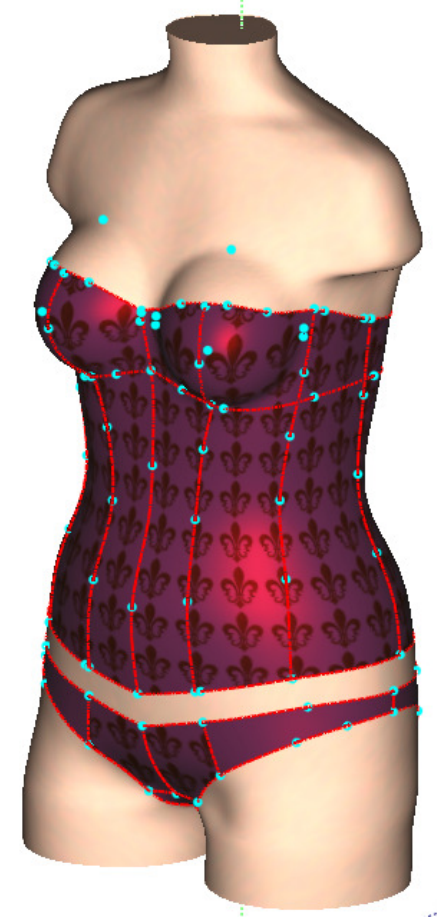

Fig. 5. 3-D corset form

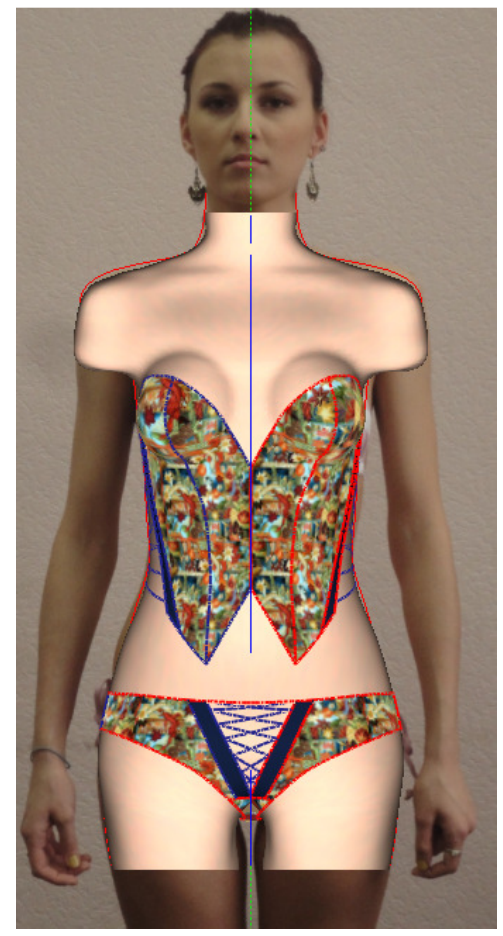

Fig. 6. 3-D corset form on the adjust mannequin 
Ready-to-use 3-D model may be easily transformed into flat patterns (cutting details) with the help of adjacent automated algorithm.

\section{Conclusions}

Developed theoretical essentials of corset ware mannequin design allow to create 3-D forms of individual and typical figures with a detail examination of breast, which is very important in corset ware design, and in the other branches, e.g. in the aesthetic medicine.

As a result of theoretical researches and their practical realization, the software program is created which gives the opportunity to design corset ware models for the individual customer using two photographs and 8 size features, to estimate aesthetic model characteristics and to obtain detail scanning. It gives the opportunity to organize customer orders to manufacture corset ware through the Internet.

\section{References}

[1] http://www.lectra.com/

[2] http://www.gerbertechnology.com/

[3] http://www.optitex.com/

[4] http://www.human-solutions.com/

[5] http://www.marvelousdesigner.com/

[6] Kornilova, N.; Balandina, G.; Gorelova, A.: CAD of corset ware in 3-D, Proceedings of Higher Education Institutions. Technology of Light Industry Vol. (2008) № 1, pp.40-44. 